\title{
¡A Abre los ojos! Un proyecto de mejora educativa para la prevención de drogas en adolescentes ${ }^{1}$
}

\section{Open your eyes! A school improvement project for the prevention of drug abuse in teenagers}

\author{
Cecilia Ma AZORÍN ABELLÁN \\ Universidad de Murcia
}

Recibido: Junio 2014

Evaluado: Septiembre 2014

Aceptado: Octubre 2014

\section{Resumen}

La Educación para la Salud (EpS) es un tema transversal del currículo que adolece de propuestas didácticas novedosas para la intervención pedagógica. El objetivo del trabajo que se presenta es poner en marcha un plan de mejora para la prevención de drogadicciones en adolescentes. Para ello, se ha trabajado con un total de 142 estudiantes de $3^{\circ}$ de Educación Secundaria Obligatoria (ESO) con edades comprendidas entre los 14 y los 16 años que cursan estudios en el Instituto "Francisco Salzillo" de la localidad de Alcantarilla (Murcia). Concretamente, este artículo da luz al Proyecto ¡Abre los ojos!, que forma parte del Plan de Acción Tutorial (PAT) y del Plan de Mejora para la Prevención de Drogas (PMPD) propuesto desde el Departamento de Orientación. Se exponen ad hoc las actividades implementadas durante las 3 sesiones trabajadas con cada uno de los 6 grupos-clase escolarizados en este nivel. Haciendo uso de la reflexión-acción, el alumnado ha desarrollado una conciencia crítica acerca de los riesgos que entraña para la salud el consumo de drogas. Asimismo, mediante la técnica de grupos de discusión los discentes han realizado un interesante debate cuyas ideas han sido organizadas en torno a tres aspectos clave: causas por las que se empieza a consumir, cómo evitar caer en las drogas, y alternativas de ocio y tiempo libre para una vida saludable. Finalmente, se especifica la necesidad de abordar tareas de prevención en los centros educativos así como de facilitar información y de resolver las inquietudes de los jóvenes acerca de esta temática.

Palabas clave: proyecto de mejora, drogas, prevención, adolescentes, educación secundaria.

\begin{abstract}
Education for Health is a cross-curricular theme that lacks innovative teaching suggestions for pedagogical intervention. The aim of the work presented is to implement an improvement plan for the prevention of drug addiction in adolescents. To do this, we worked with a total of 142 students from 3rd Compulsory Secondary Education (ESO) aged between 14 and 16 years
\end{abstract}

${ }^{1}$ Este trabajo se ha realizado dentro de una beca de investigación con apoyo financiero. 
studying in the "Francisco Salzillo" Institute of Alcantarilla (Murcia). Specifically, this article gives light to the Project Open your eyes! Which is part of the Tutorial Action Plan and proposed in the Department Improvement Plan for Drug Prevention Guide. Ad hoc activities during the 3 sessions worked with each of the 6 groups-school class at this level are presented. Using reflection-action, the students developed a critical awareness of the risks to health drug. Also, using the technique of focus groups learners made an interesting debate whose ideas have been organized around three key issues: reasons why you start to consume, how to avoid falling into drugs, and alternative recreational free time for a healthier life. Finally, it specifies the need to address prevention efforts in schools and to provide information and resolve the concerns of young people about this subject.

Keywords: improvement project, drugs, prevention, youth, secondary education.

La Educación para la Salud (EpS) es un tema transversal del currículo que adolece de propuestas didácticas novedosas para la intervención pedagógica. En este sentido, el artículo que aquí se presenta aborda la importante tarea de la prevención en el ámbito educativo, pues desde la organización escolar se ha de colaborar en la formación de las futuras generaciones y en la información acerca de los temas que son básicos para la calidad de vida de la persona. Entre ellos, la EpS aborda aspectos que tienen que ver con la educación sexual y con la prevención de drogas, contenidos catalogados como transversales. Por su parte, Becoña (2007) considera que la prevención en la escuela tendría que hacerse en una asignatura específica, dado que la transversalidad que caracteriza a esta materia hace que no tenga la intensidad que se necesita para surtir el efecto deseado. Asimismo, Segovia y Carvalho (2011) explican que el fenómeno de las drogas es una problemática que debe ser abordada de manera interdisciplinar y, por ende, transversal.

A este respecto, es prioritario que la EpS comprometa a toda la institución educativa para el fortalecimiento de una escuela sana en la que no solo se transmitan conocimientos sino que exista coherencia en la relación teórico-práctica (Vega y Aramendi, 2013). Desde esta perspectiva, se aboga por el desarrollo de unas nociones y unas competencias básicas en el discente, lo que convendría llamar Educación para la Vida y su integración en el aula.

Por otro lado, en el entorno escolar se aprecian diversidad de opiniones sobre la EpS. Para Alcántara (2011), debe tratarse la importancia de una correcta EpS en todos los niveles educativos, que propicie el desarrollo de personas conscientes de su propio bienestar y cuidado personal. Según González (2008), la EpS es una "asignatura pendiente” en la escuela. No obstante, López, Martínez y Fernández (2007) advierten que en la acción pedagógica impartida por los profesionales dedicados a la enseñanza, la EpS ha cobrado relevancia en los últimos años. En esta línea, Pérez (2006) admite que resulta interesante la puesta en práctica de programas sobre EpS, máxime si en ellos se incluyen las Tecnologías de la Información y la Comunicación (TIC) como forma de innovación.

Atendiendo a estas consideraciones, para llevar a cabo una EpS de calidad, los centros educativos deben contar con recursos suficientes que sean aplicables en el aula, 
y precisamente en esta dirección camina el proyecto ¡Abre los ojos! al que haremos alusión más adelante.

\section{La prevención de drogas desde la educación}

La adolescencia es una etapa de la vida favorable para probar las drogas, dado que la curiosidad por un lado, y la dificultad para enfrentarse a los problemas emocionales por otro, dejan constancia de la rebeldía propia de este momento (Villatoro, MedinaMora, Rojano, Fleiz, Bermúdez, Castro y Juárez, 2002). Así pues, el problema de las drogas se manifiesta primeramente en la adolescencia con el consumo de tabaco y alcohol, y al final de este período e inicio de la adultez evoluciona hacia patrones más regulares y abusivos (Gómez, Luengo y Romero, 2002).

En consecuencia, la drogodependencia es un problema que afecta a la sociedad. Por tanto, es ineluctable el desarrollo de programas y de acciones para la prevención de drogas desde el contexto educativo que incidan en la población juvenil (Banderas, Martínez y Romo, 2010; De Vincenzi y Bareilles, 2011). De este modo, la educación ha de ser vista como un instrumento de capacitación para la prevención de drogas pero también como herramienta para la promoción de una vida saludable. Desde la escuela, se pueden construir Ambientes Escolares Preventivos y utilizar uno de los instrumentos principales contra el consumo de drogas, que es precisamente la prevención (Saldarriaga, 2001; Chorén, Gavidia y Aguilar, 2010).

En los últimos años se han realizado esfuerzos para llevar a cabo una intervención universal en materia de drogadicción asumiendo la importancia de acometer proyectos de prevención de drogas en la comunidad educativa (Gómez, 1997). Sin embargo, en opinión de García (1998) “desde el punto educativo para la salud, los discursos elaborados sobre las drogas solo adquieren sentido a partir de sus efectos sociales y personales” (p. 59).

En la actualidad, debe darse un impulso decidido a la investigación en el campo de la prevención de drogas (Alonso, 2005). En esta dirección, los centros educativos han de programar tareas para la prevención temprana de drogodependencias, siendo ciertamente necesario el desarrollo de propuestas que informen a los adolescentes de los riesgos que entraña el consumo de drogas. Este tipo de programaciones han de tener en cuenta que mediante la prevención se intenta evitar que ocurran sucesos no deseables, poniendo especial acento en la instrumentación para el quehacer diario (González, Barca y Seijas, 2002).

Por consiguiente, la prevención supone dotar y capacitar al alumnado de recursos para que pueda decidir y convivir con la presencia de las drogas (Sánchez y García, 2008). En esta intersección de caminos, se aborda la EpS como un conjunto de prácticas que apuesta por una sociedad preparada para adoptar estilos de vida saludables alejados de situaciones causantes de dependencia.

Tal y como venimos expresando, la educación es per se, una herramienta de emancipación. Desde esta percepción, De Vincenzi y Tudesco (2009) afirman que la educación es una condición necesaria para promover la salud, ya que a través de ella se 
accede a la información, se desarrollan habilidades para la vida, se identifican elecciones saludables, y se empodera a los individuos en pro de su bienestar.

Por su parte, González y Pérez (1997) entienden que la mejor forma de luchar contra la droga (o cualquier otra adicción) es evitarla mediante la prevención, una lucha que ha de comenzar en las etapas de Educación Primaria y continuar más enérgicamente en Educación Secundaria, siendo primordial la tarea de promover modos de vida alejados del consumo de drogas que prevengan de las adicciones. Según Bas (2000), la prevención de drogodependencias en Educación Secundaria se ha de integrar en las áreas curriculares.

Por otro lado, Villanueva (2010) afirma que la población está altamente sensibilizada con el perjuicio provocado por el consumo de drogas, y que los ámbitos familiar y escolar se configuran como espacios idóneos para la articulación de los programas de prevención. En nuestra opinión, familia y escuela han de postularse hacia un marco de acción conjunta que posibilite una actuación integral en el discente. A este respecto, Becoña (2000) afirma que:

La prevalencia del uso y abuso de drogas en la etapa adolescente y adultez temprana son altas. Ante la alta disponibilidad, en la actualidad los adolescentes tienen que aprender a convivir con las drogas, tomando decisiones sobre su consumo o la abstinencia de las mismas. El proceso de socialización con la familia, amigos, escuela y medios de comunicación es importante en ello (p.25).

En síntesis, el propósito de la prevención es educar para la experiencia vital, lo que implica el desarrollo de hábitos, actitudes y valores positivos hacia la salud que propicien el cambio y la sensibilización. Desde este enfoque, para que el éxito de la prevención sea una realidad consolidada deben ponerse en marcha proyectos educativos que proporcionen conocimientos y creencias contrarias al consumo de drogas. En relación con ello, el centro educativo es el escenario idóneo para la implementación de tales experiencias, ya que éste actúa como agente socializador primario para la intervención preventiva (Moral, Ovejero, Sirvent y Rodríguez, 2005).

Por último, diversos estudios recientes aportan avances en cuanto a la evaluación de programas de prevención de drogas en adolescentes y ponen su atención en las características que éstos han de tener para ser eficaces (Gázquez, García y Espada, 2009; Rodríguez, Díaz, Gutiérrez, Guerrero y Gómez-Maqueo, 2011; Lorence, Hidalgo, Jiménez y Antolín, 2012). Indudablemente, todos ellos contribuyen con sus acciones a construir conocimiento y mejoran la EpS, aportando estándares de calidad a los procesos de prevención de drogas desde la educación.

\section{El profesorado y los programas de prevención de drogadicciones}

La formación de los docentes es un aspecto esencial para el éxito y la eficacia de las propuestas relacionadas con la prevención de drogadicciones. Desde esta perspectiva, el bagaje formativo del profesorado en materia de EpS es un pilar básico para la promoción de la salud en la organización escolar. Sin embargo, la escasa formación del 
profesorado sobre esta temática es, en ocasiones, uno de los principales inconvenientes para la prevención de drogas (Villanueva, 2012).

Acuñando la idea del colectivo docente como pieza clave en el marco de la EpS, se ha de tener en cuenta que una de sus principales misiones es la de formar a futuros ciudadanos que sean capaces de participar, de asumir responsabilidades, de tomar decisiones y de integrarse con normalidad en la sociedad sin necesidad de consumir drogas. Así pues, el docente no puede enfrentarse solo a tan difícil tarea sino que requiere del apoyo y de la cooperación de la sociedad en su conjunto.

Por otra parte, cabe reseñar que el Instituto de Educación Secundaria (IES) Francisco Salzillo colabora con el Grupo de Investigación EDUIN (Educación Inclusiva: Escuela para Todos) de la Universidad de Murcia en el desarrollo de planes de mejora. En esta dirección, desde el Departamento de Orientación del centro se realizan anualmente diversas actividades en el marco del Plan de Acción Tutorial (PAT). Es en este contexto donde nace el Plan de Mejora para la Prevención de Drogas (PMPD), creado con la intención de dar a conocer al alumnado de $3^{\circ}$ de ESO las consecuencias devastadoras que pueden derivarse del coqueteo con estas sustancias en la adolescencia. Expresamente, uno de los objetivos del PAT es informar al alumnado sobre los riesgos que entrañan las drogas, los tipos de drogas que hay, y sus efectos para la salud.

El Proyecto ¡Abre los Ojos! da muestra de la implicación del profesorado con este tópico y constituye un referente que visibiliza la apertura del propio centro hacia las necesidades de la sociedad, su compromiso con la EpS y más concretamente, con la campaña de prevención en materia de drogas que se acomete a través de esta experiencia. De manera general, la enseñanza de hábitos saludables dentro del ámbito educativo (y su correspondiente acción preventiva en materia de adicciones con sustancias nocivas como las drogas) precisa de un tratamiento contextual. Desde esta panorámica, el citado proyecto atiende a una realidad que está vinculada estrechamente con el escenario geosocial del que emana la información y en el que se inserta la propuesta.

En esta línea, para el diseño del plan de mejora y de las actividades, se tuvieron en cuenta las recomendaciones pedagógicas de Ferreres (2001) acerca de cómo abordar actuaciones relacionadas con la prevención de drogas:

- Prevención: es necesario que la prevención de drogas en los centros escolares se trate preferentemente en las horas dedicadas a las sesiones de tutoría de manera transversal.

- Capacitación: respecto a la capacitación de los profesores para impartir esta temática, se incide en la necesidad de contar con más información y materiales de apoyo para dar las clases.

- Continuidad: el tema de las drogas suele trabajarse en los centros de manera irregular, inconstante y desigual, aspecto que debiera dar lugar a una profunda reflexión por parte de la comunidad educativa. 
- Acción: en relación con las actuaciones que los centros educativos podrían abordar destacan, entre otras: aportar información, organizar charlas con expertos, visitar centros especializados, realizar campañas informativas/formativas para padres y alumnos, trabajar la toma de decisiones, discusión y debate.

- Y Recursos: el material a utilizar en las sesiones de prevención de drogodependencias debería ser manipulativo, motivador, con estudio de casos, aplicable directamente en la clase, y con soportes visuales.

Al hilo de estas sugerencias, el trabajo de Talavera y Gavidia (2007) recoge opiniones del profesorado respecto a la implementación de la EpS en la praxis escolar, y establece como conclusiones (1) la falta de tiempo para llevar a cabo actividades relacionadas con este contenido debido a que las programaciones docentes ya están muy ajustadas, y (2) la necesidad de reconocimiento y de apoyo al profesorado, puesto que las actividades relacionadas con la salud tienen que diseñarse fuera del horario escolar (tiempo que no es reconocido por la Administración). Otro aspecto que suele obstaculizar la práctica es (3) la descoordinación entre el profesorado, lo que dificulta la transversalidad de la propia EpS, y (4) la escasez de materiales y de recursos educativos acerca de este tópico.

Por último, asumiendo el reto que supone la realización de actividades relacionadas con la EpS, se propone a continuación un material didáctico original listo para implementar en el aula cuya finalidad es la prevención de drogas en adolescentes desde una perspectiva teórico-práctica.

\section{Objetivos}

Los objetivos que se pretenden conseguir son los siguientes:

1. Poner en marcha en el centro un plan de mejora para la prevención de drogadicciones en adolescentes.

2. Diseñar e implementar el Proyecto Piloto ¡Abre los Ojos!, dirigido a la prevención de drogas en el alumnado de $3^{\circ}$ de ESO.

\section{Metodología}

Se ha llevado a cabo una investigación-acción contextual, con un diseño descriptivo voluntario (sin ánimo de representatividad ni de generalización de los resultados) a través del cual se expone una experiencia piloto que puede ser ejemplificante para la comunidad educativa.

El Proyecto ¡Abre los Ojos! es una iniciativa emprendida por el Departamento de Orientación del IES Francisco Salzillo en el marco del Plan de Mejora para la Prevención de Drogas (PMPD) incorporado en la Programación General Anual (PGA) del centro. Durante el desarrollo del mismo, se ha trabajado una metodología de tipo participativa y activa, implicando activamente al alumnado para la adquisición de una 
actitud crítica hacia las drogas y la asunción de los peligros que entraña su consumo. Para ello, se ha propiciado la interacción, la generación de debate y la realización de actividades en grupo, favoreciendo el intercambio de ideas de un modo dinámico y abierto a los propios intereses de los educandos.

¡Abre los Ojos! se trata de una propuesta informadora y formadora, pues a través de ella se desarrolla una conciencia y una sensibilización de aprendizaje para la vida. Asimismo, se ha potenciado el uso de las TIC para la presentación de la información en las diferentes sesiones. A este respecto, se ha tenido en cuenta que las aulas de hoy día están formadas por nativos digitales que demandan el uso de la tecnología en el proceso de enseñanza y aprendizaje. Precisamente, las generaciones actuales se posicionan cada vez más a favor del formato digital frente al impreso. Por tanto, la utilización de la tecnología en las propuestas didácticas permite captar mejor la atención del discente, y consigue una mayor motivación a la hora de acometer las tareas educativas (Arnaiz y Azorín, 2012; Tarango, Romo-González, Murguía-Jáquez y Ascensio-Baca, 2014).

\section{Destinatarios}

El Proyecto ¡Abre los Ojos! se ha implementado en horario de Tutoría con 6 grupos-clase del IES Francisco Salzillo, ubicado en la localidad de Alcantarilla (Murcia). Concretamente, la experiencia ha contado con la participación de 142 adolescentes que cursan enseñanzas en $3^{\circ}$ de ESO, incluyendo aquellos que se encuentran dentro del Programa de Diversificación Curricular (PDC) y del Programa de Cualificación Profesional Inicial (PCPI), con edades comprendidas entre los 14 y los 16 años.

\section{Páginas web de interés relacionadas}

La propuesta ¡Abre los Ojos! comienza con la exposición al alumnado de una presentación audiovisual. Para la elaboración de este material se ha consultado el Plan Nacional sobre Drogas (http://www.pnsd.msc.es) del Ministerio de Sanidad, Servicios Sociales e Igualdad. También ha sido de gran ayuda la información contenida en la web de la Asociación Proyecto Hombre (http://proyectohombre.es/), encargada de la prevención y tratamiento de las drogodependencias y otras adicciones, así como los contenidos que alberga el espacio virtual de la Fundación de Ayuda contra la Drogadicción (FAD) (http://www.fad.es/).

Otro entorno web interesante de obligada referencia y consulta es el que contiene el espacio "Construye tu Mundo" (http://www.construyetumundo.org/), que presenta un programa de prevención de conductas de riesgo en el que se proponen diversas actividades para trabajar sobre las drogas, en su mayoría dirigidas al alumnado de Educación Primaria y de Educación Secundaria. En esta plataforma, la información aparece desglosada por cursos y temas de interés (por ejemplo: problemas con las drogas, reafirmación de la personalidad, trabajo de la autoestima...), y se ofrece un amplio elenco de materiales didácticos que están a disposición del profesorado con un solo clic del ratón. 
A partir de esta revisión de contenidos y fuentes de información digital, se ha elaborado un material didáctico inédito (Presentación audiovisual del Proyecto ¡Abre los Ojos!) que esperamos sea de utilidad para su desarrollo en Educación Secundaria por parte de los Profesores Tutores, Orientadores, Pedagogos y Profesores Técnicos de Servicio a la Comunidad (PTSC).

\section{El Proyecto ¡Abre los Ojos!}

\section{Presentación}

El proyecto ¡Abre los Ojos! se llevó a cabo durante 3 sesiones de 55 minutos con cada uno de los grupos participantes en un marco temporal que ocupó 3 semanas. En la siguiente tabla (tabla 1), se expone información relativa a la organización de las sesiones por contenidos y actividades.

\begin{tabular}{|c|c|c|}
\hline & Contenidos & Actividades \\
\hline $\begin{array}{c}1^{\mathrm{a}} \\
\text { Sesión }\end{array}$ & $\begin{array}{l}\text {-Tipos de drogas: depresivas, } \\
\text { estimulantes y alucinógenas. } \\
\text {-Tabaco, alcohol y derivados del } \\
\text { Cannabis. }\end{array}$ & $\begin{array}{l}\text {-Cuestionario de evaluación inicial. } \\
\text {-Exposición teórico-informativa. } \\
\text {-Visualización y valoración crítica de los vídeos. } \\
\text {-Síntesis y comentario de lo aprendido. }\end{array}$ \\
\hline $\begin{array}{c}2^{\mathrm{a}} \\
\text { Sesión }\end{array}$ & $\begin{array}{l}\text { - Cocaína, heroína y drogas de } \\
\text { síntesis. }\end{array}$ & $\begin{array}{l}\text {-Repaso de los contenidos de la sesión anterior } \\
\text { (evaluación continua). } \\
\text {-Cuestionario de evaluación inicial. } \\
\text {-Exposición teórico-informativa. } \\
\text {-Visualización y valoración crítica de los vídeos. } \\
\text {-Síntesis y comentario de lo aprendido. }\end{array}$ \\
\hline $\begin{array}{c}3^{\mathrm{a}} \\
\text { Sesión }\end{array}$ & $\begin{array}{l}\text {-Formas de decir NO a las drogas. } \\
\text {-Causas por las que se empieza a } \\
\text { tomar drogas. } \\
\text {-Cómo evitar el consumo de drogas. } \\
\text {-Alternativas de ocio y tiempo libre } \\
\text { alejadas de prácticas nocivas para la } \\
\text { salud. }\end{array}$ & $\begin{array}{l}\text {-Repaso de los contenidos de las sesiones } \\
\text { anteriores (evaluación continua). } \\
\text {-Realización del ejercicio ¡Formas de decir NO a } \\
\text { las drogas! } \\
\text {-Formación de los grupos de discusión. } \\
\text {-Trabajo de las cuestiones planteadas mediante la } \\
\text { generación de ideas en el debate de los pequeños } \\
\text { grupos. } \\
\text {-Puesta en común de las aportaciones de los } \\
\text { grupos de discusión al gran grupo. } \\
\text {-Cuestionario de evaluación final. }\end{array}$ \\
\hline
\end{tabular}

Tabla 1. Sesiones del Proyecto ¡Abre los Ojos!

Atendiendo a los estudios de Botvin (1995) y de Alfonso, Huedo-Medina y Espada (2009), los esfuerzos en prevención primaria han de estar dirigidos a los adolescentes para reducir el consumo de tabaco, alcohol y cannabis, ya que es habitual la incidencia de de estas drogas entre los jóvenes. Considerando estos indicios, la experiencia 
didáctica que se propone abarca un mayor detenimiento en estas drogas con la intencionalidad de incidir más en ellas debido a su prevalencia en edades tempranas.

\section{Recursos}

En las dos primeras sesiones se abordaron contenidos teórico-informativos y se trabajó con el material audiovisual diseñado. La presentación utilizada por la pedagoga durante las exposiciones teóricas se encuentra disponible para su consulta y descarga en la dirección http://cmariaazorin.wix.com/abrelosojos (figura 1).

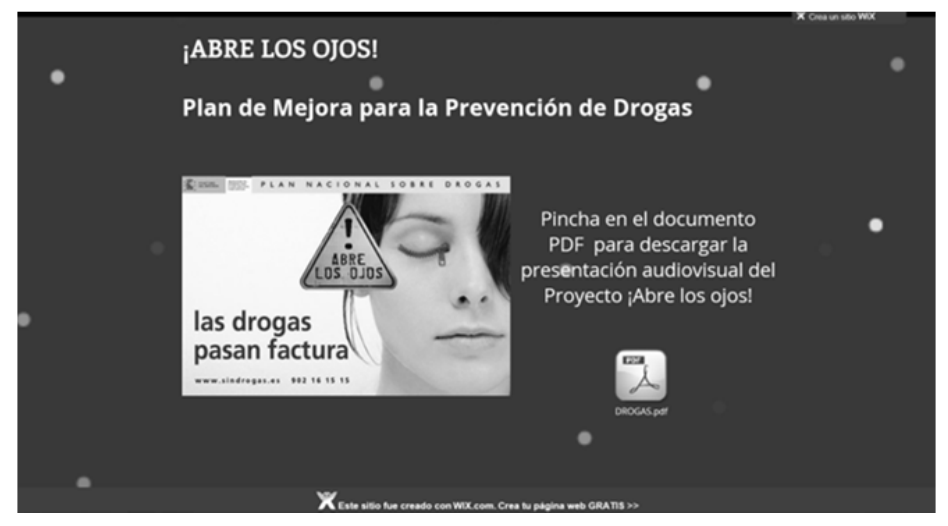

Figura 1. Captura de la página principal del Proyecto ¡Abre los ojos! en la red

Asimismo, cabe destacar que la imagen con la que comienza este recurso didáctico pertenece a la campaña "Abre los ojos, las drogas pasan factura”, propuesta en el año 2003 por la Delegación del Plan Nacional sobre Drogas del Ministerio del Interior español.

\section{Actividades}

La experiencia empezó con un proceso de evaluación inicial (al inicio de la primera y segunda sesión) en el que se utilizó un cuestionario tipo test para delimitar los conocimientos previos que tenían los discentes sobre las drogas. Se realizó, ad hoc, una adaptación del cuestionario ¿Qué sabes de las drogas? del Programa "Mano a Mano" del Gobierno de La Rioja. En la dirección http://manoamano.riojasalud.es/bdi_tests.html puede consultarse el banco de instrumentos de este programa. El cuestionario original se ubica en el dominio http://manoamano.riojasalud.es/pdfs/test_quesabesdelasdrogas.pdf.

En la primera sesión se trabajó la parte que contiene 9 ítems (a contestar verdaderofalso) en los que se incluyen aspectos relacionados con el alcohol, el tabaco y los derivados del cannabis (tabla 2): 


\begin{tabular}{ll}
\hline \multirow{3}{*}{ Alcohol } & 1. Es una droga que estimula el sistema nervioso (V-F) \\
& $\begin{array}{l}\text { 2. El alcohol disminuye los reflejos (V-F) } \\
\text { 3. Al principio te despierta y anima, aunque luego te adormece (V-F) }\end{array}$ \\
\hline \multirow{3}{*}{ Tabaco } & $\begin{array}{l}\text { 4. Hay que fumar mucho para notar los efectos (V-F) } \\
\text { 5. Dificulta la circulación de la sangre y produce enfermedades del aparato } \\
\text { respiratorio (V-F) } \\
\text { 6. El tabaquismo es una costumbre más que una adicción (V-F) }\end{array}$ \\
\hline \multirow{2}{*}{ Cannabis } & $\begin{array}{l}\text { 7. El uso continuado provoca apatía y falta de concentración (V-F) } \\
\text { 8. No provoca ninguna complicación en el sistema respiratorio (V-F) } \\
\text { 9. Tan solo produce dependencia física (V-F) }\end{array}$ \\
\hline
\end{tabular}

Tabla 2. Cuestionario de evaluación inicial primera sesión (Adaptación del Programa "Mano a Mano”)

Por otro lado, la continuación del cuestionario de evaluación inicial utilizado en la segunda sesión está formado por 12 ítems, también con elección de respuesta verdadero-falso. En este caso, los contenidos están referidos a la cocaína, el éxtasis, los alucinógenos y la heroína (tabla 3):

\begin{tabular}{ll}
\hline \multirow{2}{*}{ Cocaína } & $\begin{array}{l}\text { 10. Su consumo causa euforia y excitación (V-F) } \\
\text { 11. Puede hacer que la persona sea fácilmente irritable (V-F) } \\
\text { 12. Su consumo no repercute en los hábitos de sueño y alimentación (V-F) }\end{array}$ \\
\hline \multirow{2}{*}{ Éxtasis } & $\begin{array}{l}\text { 13. Es una droga inocua, no provoca daños en la salud (V-F) } \\
\text { 14. Todas las pastillas están compuestas de las mismas sustancias (V-F) } \\
\text { 15. Suele conocerse también como droga de síntesis (V-F) }\end{array}$ \\
\hline Alucinógenos & $\begin{array}{l}\text { 16. Sus efectos están en función, solamente, de la dosis consumida (V-F) } \\
\text { 17. Uno mismo puede controlar sus efectos y detenerlos (V-F) } \\
\text { 18. Existe riesgo de episodios de pánico y accidentes cuando se está bajo sus } \\
\text { efectos (V-F) }\end{array}$ \\
\hline Heroína & $\begin{array}{l}\text { 19. Produce ansiedad y nerviosismo cuando se pasan sus efectos (V-F) } \\
\text { 20. Aumenta los deseos sexuales (V-F) } \\
\text { 21. Mezclada con tranquilizantes, con metadona o alcohol no da problemas (V- } \\
\text { F) }\end{array}$ \\
\hline
\end{tabular}

Tabla 3. Cuestionario de evaluación inicial segunda sesión (Adaptación del Programa "Mano a Mano”)

Al terminar la experiencia, en la tercera sesión se volvió a pasar el cuestionario a modo de evaluación final juntando los 21 ítems de los que consta el instrumento adaptado. Seguidamente, se detalla el modus operandi seguido durante la experiencia.

- Exposiciones teórico-informativas: en la primera sesión, se trabajaron los tipos de drogas que existen (depresivas, estimulantes y alucinógenas), y se profundizó en las siguientes: tabaco, alcohol y derivados del Cannabis. En la segunda sesión se abordaron otros tipos de drogas más fuertes como son la cocaína, la heroína y las drogas de síntesis, y se habló de los mitos y realidades de los contenidos programados. Esta información se encuentra disponible para su consulta en la dirección web del proyecto facilitada anteriormente. 
- Visualización y valoración crítica de los vídeos: tanto en la primera como en la segunda sesión se llevó a cabo el visionado de distintos vídeos que acompañaban el discurso de la pedagoga con la intencionalidad de que el alumnado asumiera la dureza de las drogas desde una perspectiva realista de las mismas. Para ello, se proyectaron diferentes escenas de una selección de sucesos, documentales, noticias y cortometrajes. Con estos visionados (que se encuentran a disposición del lector en la web del Proyecto ¡Abre los ojos!) se pretendió sensibilizar e impactar acerca del riesgo que supone el consumo de sustancias.

- Síntesis y comentario de lo aprendido: al finalizar cada una de las sesiones, se realizó una síntesis y comentario de lo aprendido. En estos momentos, se dio voz al alumnado para que expresara sus inquietudes y para que preguntara las dudas generadas tras el discurso en el aula. Se procuró así que los discentes reflexionaran sobre su propio conocimiento y sobre los contenidos de las actividades propuestas, haciéndoles partícipes de su proceso de aprendizaje, y construyendo así una verdadera conciencia crítica en ellos para una toma de decisiones futura de manera responsable.

- Ejercicio ¡Formas de decir NO a las drogas!: en esta actividad se dan pautas al alumnado para afrontar situaciones en las que las drogas pueden pasar por su vida cotidiana, y se enseña cómo responder ante la insistencia de otras personas que incitan al consumo. Algunas de las propuestas fueron las siguientes:

- Di ¡no! sin más.

- Justifica tu negativa.

- Actúa como un disco rayado: no, no, no... Este es un recurso de desgaste que se utiliza hasta que dejan de insistir.

- Devuelve el mismo argumento (técnica del espejo).

- Puedes darle la razón a la otra persona pero luego hacer lo que tú quieras (banco de niebla).

- Enfréntate con ironía/sarcasmo (es importante que te muestres fuerte ante la tentativa).

- Sugiere otras alternativas que no impliquen el consumo de drogas.

- Sal de la situación, no aceptes la presión del grupo ni hagas lo que no quieres. 
- Grupos de discusión: se trata de una técnica de gran utilidad para la obtención de información cualitativa. Estos grupos (figura 2) han estado formados por 4-6 personas (dependiendo del número de estudiantes de cada clase). Esta actividad ha permitido conocer las valoraciones y las percepciones del alumnado acerca del tema objeto de estudio: las drogas.

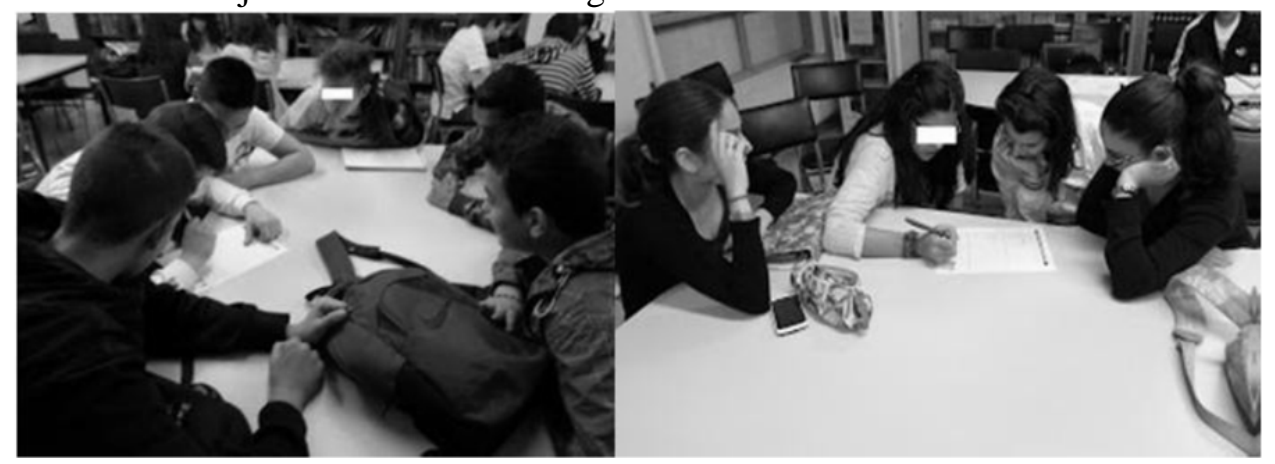

Figura 2. Grupos de discusión formados por estudiantes de $3^{\circ}$ de ESO

Para la realización de la tarea se facilitó al alumnado una ficha (que debía ser cumplimentada de manera grupal) con información acerca de las causas por las que se empieza a tomar drogas, cómo evitar el consumo, y alternativas de ocio y tiempo libre alejadas de prácticas nocivas para la salud (tabla 4).

\begin{tabular}{l|l}
\hline \multicolumn{2}{c}{ GRUPOS DE DISCUSIÓN SOBRE DROGAS } \\
\hline \multirow{4}{*}{$\begin{array}{l}\text { Causas por las que se } \\
\text { empieza a consumir } \\
\text { droga }\end{array}$} & $\begin{array}{l}\text { Como vía de escape a los problemas, afán de huir de la realidad, } \\
\text { problema derivado de un fracaso, mala etapa, déficit de afecto, } \\
\text { baja autoestima, curiosidad, nuevas sensaciones, aburrimiento, } \\
\text { soledad, depresión, familiares o amigos que consumen, } \\
\text { desinformación, publicidad engañosa en los medios de } \\
\text { comunicación, por ser aceptado en el grupo de amigos (presión } \\
\text { del grupo). }\end{array}$ \\
\hline $\begin{array}{l}\text { Formas de evitar las } \\
\text { drogas }\end{array}$ & $\begin{array}{l}\text { No salir con malas amistades, tener una buena autoestima, no } \\
\text { aceptar la presión del grupo, hacer actividades que impliquen un } \\
\text { estilo de vida saludable alejado del consumo de drogas, estar } \\
\text { informado del riesgo que entraña el consumo de drogas para no } \\
\text { caer en posibles tentaciones. }\end{array}$ \\
\hline $\begin{array}{l}\text { Alternativas de ocio y } \\
\text { tiempo libre saludables }\end{array}$ & $\begin{array}{l}\text { Hacer deporte, ir a la playa, ir a la montaña, ir al cine, realizar } \\
\text { alguna actividad de voluntariado, celebrar fiestas sin drogas con } \\
\text { los amigos, realizar actividades, cursos y viajes, tomar } \\
\text { fotografías, hacer música... En definitiva, todo aquello que } \\
\text { implique una alternativa y que permita el entretenimiento } \\
\text { saludable de la persona. }\end{array}$ \\
\hline
\end{tabular}

Tabla 4. Ficha de trabajo de los Grupos de Discusión sobre Drogas 
La tabla anterior recoge una síntesis de cada una de las cuestiones tratadas en los grupos de discusión que serán a su vez reflejadas en la red semántica expuesta en el apartado de resultados.

\section{Recogida y análisis de la información}

En cuanto a la recolección de la información, la pedagoga recogió en papel impreso los diferentes documentos (principalmente los cuestionarios y las fichas elaboradas por los grupos de discusión) al término de las actividades propuestas para su posterior análisis. Asimismo, las respuestas contenidas en los cuestionarios inicial y final fueron analizadas mediante el paquete estadístico SPSS en su versión 22, lo que ha permitido la obtención de los porcentajes de acierto-error asociados a los diferentes ítems. Por otro lado, la información cualitativa de la ficha rellenada por los grupos de discusión fue volcada en el software Atlas.ti versión 7.

\section{Evaluación y resultados}

La evaluación, tal y como se recoge en el apartado de actividades, se ha realizado en tres momentos: evaluación inicial (al inicio de la experiencia en la primera y segunda sesión utilizando para ello el cuestionario de evaluación inicial), evaluación continua (durante las tres sesiones se han trabajado actividades de evaluación continua, un ejemplo de esto son los ejercicios de "Mitos y Realidades" que se proponen al finalizar la explicación de cada una de las drogas), y evaluación final (con la cumplimentación del cuestionario al término de la experiencia).

Los instrumentos utilizados para la recogida de información han sido (1) el cuestionario sobre drogas, (2) la observación participante, (3) la información recogida a partir de los grupos de discusión en la ficha de trabajo, y (4) los debates generados en el aula (gran grupo) en relación con el consumo de drogas.

Los resultados del cuestionario inicial denotan que el alumnado (de forma previa a la experiencia) estaba bastante desinformado respecto a este campo de conocimiento, pues erróneamente el $40 \%$ consideraba que el alcohol es una droga que actúa como estimulante del sistema nervioso, el 35\% que el cannabis produce dependencia física pero no psicológica, y el 15\% que los efectos de los alucinógenos están en función, solamente, de la dosis consumida. Sin embargo, el $100 \%$ sabía a priori entre otras cuestiones que el consumo de alcohol disminuye los reflejos, y que la heroína produce ansiedad y nerviosismo.

Al finalizar la experiencia, respecto a los resultados del cuestionario final, el 89\% del alumnado contestó correctamente a la mayoría de las preguntas mientras que el 11\% restante cometió entre 1 y 3 errores. Estos datos son entendidos como positivos, pues en la prueba inicial la mayoría de los discentes tuvieron de media entre 5 y 8 errores. Ello denota que los grupos-clase se han mantenido atentos al mensaje transmitido durante las explicaciones teórico-prácticas captándolo adecuadamente. 
Respecto a la observación participante, la pedagoga ha valorado el grado de participación e interés mostrado por el alumnado durante la implementación de la experiencia. A este respecto, no todos los grupos han acogido del mismo modo el proyecto, pues en algunos cursos se observó que había mayor motivación que en otros, como es el caso del grupo perteneciente al PCPI, en el que el alumnado se mostró menos receptivo al discurso generado en el aula.

Por otro lado, tal y como se ha comentado con anterioridad, la información recogida en la ficha trabajada por los grupos de discusión ha sido volcada en el programa Atlas.ti, un software estadístico con el que se ha creado la red semántica que resume la opinión del alumnado acerca de cómo se empieza a consumir drogas, formas de decir no (maneras de evitar el consumo), y alternativas de ocio y tiempo libre (gráfico 1).

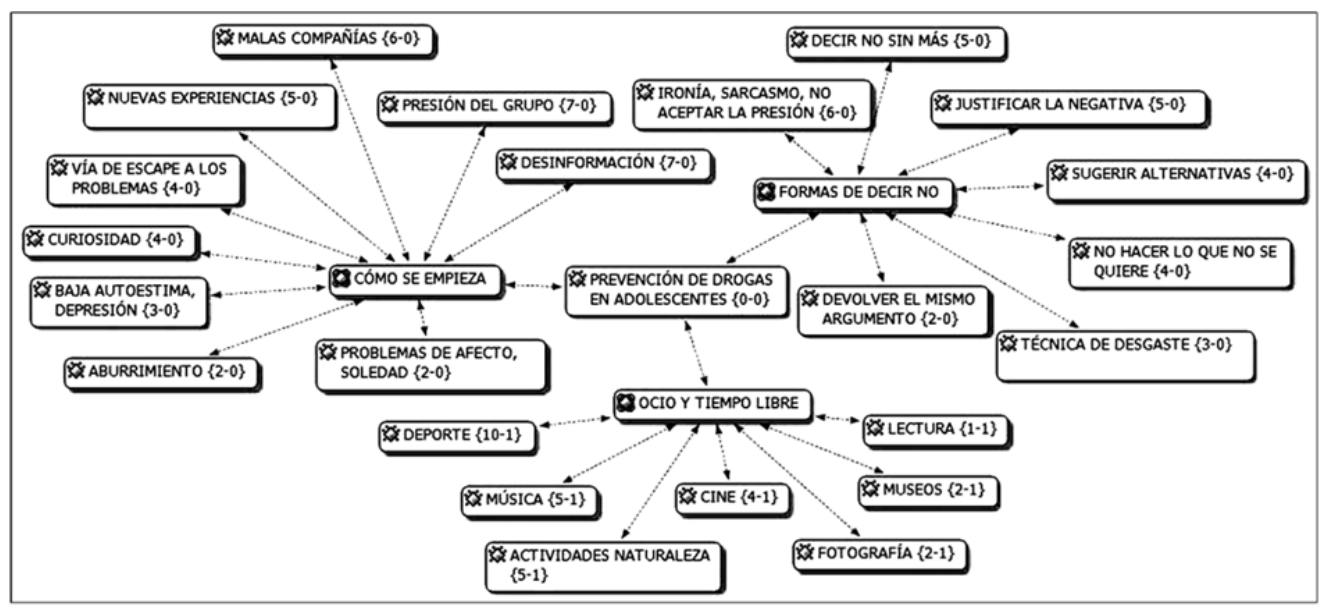

Gráfico 1. Red semántica de ideas emitidas por los Grupos de Discusión

El gráfico representado incluye los resultados derivados del análisis de la información aportada por los grupos de discusión. En su mayoría, las contestaciones coinciden con la información trabajada durante las sesiones teórico-informativas, lo que pone de manifiesto que el alumnado reproduce las ideas que le han sido transmitidas en el aula.

Acerca de cómo se empieza, Díaz-Aguado, Martínez-Arias y Ordóñez (2013) asumen que, entre las principales condiciones de riesgo para el consumo de drogas en la adolescencia, destaca la vulnerabilidad a la presión del grupo de iguales, la justificación del consumo de drogas, y la búsqueda de sensaciones asociadas al riesgo, lo que coincide con los argumentos del alumnado participante en la experiencia.

Por otro lado, los debates realizados en la puesta en común de ideas al gran grupo han dado cuenta de la participación activa de los discentes, si bien es cierto que estos estudiantes no están acostumbrados a hacer debates en el aula y mostraron ciertas reticencias, aunque finalmente aportaron de buen grado sus opiniones respecto al leitmotiv sobre el que giraba el discurso (las drogas). 


\section{Conclusiones y prospectiva}

Según los objetivos formulados en este trabajo, puede concluirse que se ha puesto en marcha de manera satisfactoria el Plan de Mejora para la Prevención de Drogas. Paralelamente, el Proyecto ¡Abre los ojos! ha sido diseñado y desarrollado con éxito. En esta dirección, durante la implementación de la propuesta ha habido una respuesta positiva por parte del alumnado de $3^{\circ}$ de ESO.

Asimismo, una de las principales conclusiones que se establece es la necesidad de abordar tareas de prevención en centros educativos, y de resolver las inquietudes de los jóvenes en torno a las drogas, estableciendo para ello un feedback y una relación cercana entre profesor-alumno.

Tras la experiencia realizada, se consideran imprescindibles las campañas e iniciativas para hablar en el aula de forma abierta con el alumnado sobre los temas que preocupan e interesan a estas edades, como es el caso de las drogas. Así pues, adquiere relevancia la difusión de experiencias didácticas novedosas para que el colectivo docente disponga de materiales y de recursos de intervención pedagógica en la literatura que faciliten su labor preventiva en el centro educativo.

En nuestra opinión, el alumnado ha de adquirir unas nociones básicas de Educación para la Vida, y entre estas enseñanzas se encuentra el tópico de las drogas. Se corrobora igualmente la importancia de acometer programas y acciones de mejora para la prevención de drogas desde el contexto escolar que tengan especial incidencia en los jóvenes, colectivo potencialmente en riesgo al que han de dirigirse los mayores esfuerzos. A continuación, haciendo balance del Proyecto ¡Abre los Ojos! se presentan las fortalezas detectadas desde la visión docente:

- La presentación de los contenidos a través de las TIC: en este trabajo adquiere interés y significación el uso de la tecnología en el aula con los nativos digitales.

- El vídeo como recurso de apoyo en el proceso de enseñanza-aprendizaje: la utilización del vídeo en las tareas educativas incrementa el nivel de motivación del alumnado.

- El desarrollo de claves para decir no a las drogas: ello conlleva otorgar pautas al discente para salir airoso de situaciones en las que puede verse comprometido, e implica el desarrollo de competencias y de habilidades para la vida, y para la construcción de su propia personalidad.

- Los grupos de discusión entre iguales: se ha observado que la utilización de esta técnica es muy adecuada a la hora de incidir en temas relacionados con la EpS, como es el caso de las drogas.

- La generación de debate: lo que permite dar voz al alumnado para expresar sus opiniones y propicia el desarrollo de ideas, la escucha atenta, y la creación de una conciencia crítica.

- El trabajo de los mitos y las realidades: es importante que el discente no aprenda por leyendas urbanas sino porque tenga a su disposición la 
información necesaria de forma veraz y contrastada por profesionales de la educación.

- La reflexión-acción a partir de interrogantes ¿por qué?, ¿cómo evitar?, ¿qué hacer?: es indispensable que el alumnado se cuestione cosas, que desarrolle su capacidad crítica y su pensamiento divergente.

En relación con las debilidades, destacan las siguientes:

- Un marco temporal escaso para la sedimentación de los contenidos: el Proyecto ¡Abre los Ojos! se ha implementado durante 3 semanas. Obviamente, se requiere una mayor dedicación dentro del calendario escolar para que el mensaje que se transmite al alumnado en el proceso de aprendizaje tenga el calado que sería deseable.

- La experiencia podría trabajarse en otros cursos y centros educativos: sería interesante valorar el impacto y el desarrollo del proyecto realizado no solo con $3^{\circ}$ de ESO sino también en otros niveles, y observar así la puesta en marcha del proyecto en diversos contextos y realidades educativas.

Para terminar, se proponen algunas sugerencias a modo de prospectiva:

- Mantener la línea de mejora emprendida para darle continuidad.

- Organizar charlas y seminarios de prevención de drogas en diferentes niveles educativos.

- Planificar visitas de expertos al centro.

- Fomentar el sentido de aula permeable, acogiendo los intereses del alumnado.

- Realizar un taller de padres y madres que lleve por título ¿Hablas de drogas con tu hijo/a? Paralelamente, sería interesante que los hermanos/as y el grupo de iguales participaran como agentes de información-mediatización de la intervención preventiva, y que en un futuro cercano se integrase a los diversos sectores sociales sobre los que interactúa directamente el alumnado, incorporando de forma activa no solo a su núcleo familiar sino también a su entorno vecinal.

- Sensibilizar al alumnado a partir de campañas informativas y publicitarias con trabajos realizados por ellos mismos que queden expuestos en las aulas y los tablones de anuncios del centro escolar.

- Y crear un buzón de sugerencias dirigidas al profesorado para que el alumnado realice a través de este y otros mecanismos sus propuestas al equipo docente.

En definitiva, existe la necesidad de abordar tareas de prevención en los centros educativos, de facilitar información, y de resolver las dudas de los jóvenes acerca de esta temática. Finalmente, en este artículo se constata que el Proyecto ¡Abre los Ojos! responde a este perfil, y que contiene un material útil para introducir la prevención de drogas en el aula de Educación Secundaria de un modo dinámico y cercano a los intereses de los discentes. 


\section{Agradecimientos}

Nuestro agradecimiento al Ministerio de Educación, Cultura y Deporte por la concesión de la Beca de Formación del Profesorado Universitario (FPU13/00580) a la que se encuentra vinculado este trabajo.

\section{Referencias bibliográficas}

ALCÁNTARA, M. D. (2011). Relevancia de la educación para la salud en nuestro alumnado. Revista de Innovación y Experiencias Educativas, 39, 1-8.

ALFONSO, J.P., HUEDO-MEDINA, T.B. y ESPADA, J.P. (2009). Factores de riesgo predictores del patrón de consumo de drogas durante la adolescencia. Anales de

Psicología, 25(2), 330-338.

ALONSO, C. (2005). La prevención del consumo de drogas en España: evolución y propuestas para la reflexión. Revista Española de Drogodependencias, 30(3-4), 367-381.

ARNAIZ, P. y AZORÍN, C.M. (2012). El edublog como herramienta de aprendizaje para todos en el entorno virtual. Didáctica, Innovación y Multimedia, 24, 1-12.

BANDERAS, C.R., MARTÍNEZ, A.J. y ROMO, T. (2010). Prevención integral del consumo de alcohol y drogas en estudiantes universitarios: una propuesta de intervención grupal. Acta Colombiana de Psicología, 13(2), 19-33.

BAS, E. (2000). Prevención de drogodependencias, en secundaria. Integración en las Áreas curriculares. Madrid: Narcea.

BECOÑA, E. (2000). Los adolescentes y el consumo de drogas. Papeles del psicólogo, 77, 25-32.

BECOÑA, E. (2007). Bases psicológicas de la prevención del consumo de drogas. Papeles del Psicólogo, 28(1), 11-20.

BOTVIN, G.J. (1995). Entrenamiento en habilidades para la vida y prevención del consumo de drogas en adolescentes: consideraciones teóricas y hallazgos empíricos. Psicología Conductual, 3(3), 333-356.

CHORÉN, S., GAVIDIA, C. y AGUILAR, R. (2010). Elaboración de un instrumento para el análisis de los programas de prevención de drogodependencias dirigidos al ámbito familiar. Didáctica de las Ciencias Experimentales y Sociales, 24, 107-121.

DE VINCENZI, A. y BAREILLES, G. (2011). Promoción de la salud y prevención escolar del consumo de drogas en contextos de vulnerabilidad social. Educación y Educadores, 14(3), 577-600.

DE VINCENZI, A. y TUDESCO, F. (2009). La educación como proceso de mejoramiento de la calidad de vida de los individuos y de la comunidad. Revista Iberoamericana de Educación, 49(7), 1-12. 
DÍAZ-AGUADO, M.J., MARTÍNEZ-ARIAS, R. y ORDÓÑEZ, A. (2013). Prevenir la drogodependencia en adolescentes y mejorar la convivencia desde una perspectiva escolar ecológica. Revista de Educación, Extraordinario, 338-362.

FERRERES, V.S. (2001). El profesorado y la prevención de las drogas: bases para la elaboración de materiales. Revista Electrónica Interuniversitaria de Formación del Profesorado, 4(2), 1-10.

GARCÍA, A. (1998). Educación para la salud y drogas: hacia un cambio de enfoque. Pedagogía Social, 1, 59-69.

GÁZQUEZ, M., GARCÍA, J.A. y ESPADA, J.P. (2009). Características de los programas eficaces para la prevención escolar del consumo de drogas. Health and Addictions, 9(2), 185-208.

GÓMEZ, A. (1997). Prevenir las drogas desde la comunidad educativa. Una propuesta desde la Educación para la Salud y el Consumo. Tabanque, 12-13, 109-129.

GÓMEZ, J.A., LUENGO, A. y ROMERO, E. (2002). Prevención del consumo de drogas en la escuela: cuatro años de seguimiento de un programa. Psicothema, 14(4), 685-692.

GONZÁLEZ, A. M., BARCA, A. y SEIJAS, S. (2002). Educación para la salud: un programa de prevención de las drogodependencias, desarrollo y crecimiento personal. Revista Gallego-Portuguesa de Psicología de la Educación, 8(6), 75-98.

GONZÁLEZ, E. y PÉREZ, P. (1997). La intervención educativa en el campo de las drogodependencias. La formación para la prevención en la escuela. Revista Complutense de Educación, 8(1), 79-101.

GONZÁLEZ, M.D. (2008). La educación para la salud: “asignatura pendiente” para la escuela. Revista de Educación, 10, 123-136.

LÓPEZ, E. J., MARTÍNEZ, A. J. y FERNÁNDEZ, E.M. (2007). Educación para la salud: Guía para la prevención del consumo de drogas en la adolescencia. Aldadis.net, 11, 18-21.

LORENCE, B., HIDALGO, M.V., JIMÉNEZ, L. y ANTOLÍN, L. (2012). Evaluación de la implementación de dos programas de prevención de drogodependencias en el sistema educativo andaluz: Dino y Prevenir para Vivir. Revista de Educación, 358, 334-356.

MORAL, M.V., OVEJERO, A., SIRVENT, C. y RODRÍGUEZ, F.J. (2005). Prevención e intervención psicosocial sobre el consumo juvenil de sustancias psicoactivas: Comparación de la eficacia preventiva de cuatro programas. Intervención Psicosocial, 14(2), 189-208.

PÉREZ, I. J. (2006). Una experiencia de innovación en educación para la salud a través de las nuevas tecnologías. Revista de Educación, 34, 917-932. 
RODRÍGUEZ, S.E., DÍAZ, D.B., GUTIÉRREZ, S.E.G., ABELARDO, J. y GÓMEZMAQUEO, E.L. (2011). Evaluación de un programa de prevención del consumo de drogas para adolescentes. Salud Mental, 34, 27-35.

SALDARRIAGA, J. (2001). Drogas, escuela y formación. Educación XXI: Revista de la Facultad de Educación, 4, 189-200.

SÁNCHEZ, A.M. y GARCÍA, A. (2008). Prevención del abuso de drogas y promoción de la salud en los jóvenes. Revista de Estudios y Experiencias en Educación, 14, 117-130.

SEGOVIA, N.S. y CARVALHO, M.F. (2011). Los espacios escolares para la prevención de la drogodependencia: concepción de directivas de escuelas secundarias. Revista Latinoamericana Enfermagem, 19, 782-788.

TALAVERA, M. y GAVIDIA, V. (2007). Dificultades para el desarrollo de la educación para la salud en la escuela. Opiniones del profesorado. Didáctica de las Ciencias Experimentales y Sociales, 21, 119-128.

TARANGO, J., ROMO-GONZÁLEZ, J.R., MURGUÍA-JÁQUEZ, L.P. y ASCENCIO-BACA, G. (2014). Uso y acceso a las TIC en estudiantes de escuelas secundarias públicas en la ciudad de Chihuahua, México: inclusión en la didáctica y en la alfabetización digital. Revista Complutense de Educación, 25(1), 133-152.

VEGA, A. y ARAMENDI, P. (2013). Alcohol en la Educación Secundaria Obligatoria: desde la preocupación por la borrachera al compromiso educativo de los centros escolares. Revista Española de Drogodependencias, 38(3), 251-262.

VILLANUEVA, C. (2010). Resultados de un programa de prevención de drogas en el cambio de actitudes del alumnado. Enseñanza \& Teaching, 28(1), 157-183.

VILLANUEVA, C. (2012). Influencia de la familia en la prevención de drogas. Revista Electrónica de Investigación y Docencia, 7, 176-193.

VILLATORO, J., MEDINA-MORA, M.E., ROJANO, C., FLEIZ, C., BERMÚDEZ, P., CASTRO, P. y JUÁREZ, F. (2002). ¿'Ha cambiado el consumo de drogas de los estudiantes? Medición otoño del 2000. Salud Mental, 25(1), 43-54.

\section{Correspondencia con la autora}

Cecilia Ma AZORÍN ABELLÁN

Facultad de Educación

Universidad de Murcia, Campus de Espinardo

30100 Murcia (España)

e-mail: cmaria.azorin@um.es 